\title{
Collision Resolution Based on Independent Component Analysis
}

\author{
Xi Chen, Qinyu Zhang \\ Communication Engineering Research Center \\ Harbin Institute of Technology \\ Guangdong, P.R.China \\ zqy@hit.edu.cn
}

\author{
Ye Wang \\ Communication Engineering Research Center \\ Harbin Institute of Technology \\ Guangdong, P.R.China
}

\begin{abstract}
This paper proposed a retransmission scheme based on the blind separation method, named independent component analysis(ICA), to resolve the collision problem in random access wireless network and a new method to devise the Identification(ID)sequences which can resolve the problems of ICA and help to reduce the length of ID sequences. The proposed method can work effectively under the fast-varying and slow-varying channels from the simulation results.
\end{abstract}

Keywords- blind separation, retransmission scheme, random access

\section{INTRODUCTION}

In this epoch of the eruption of information, the demand of the communication services is increasing dramatically, so the traditional fixed bandwidth allocation schemes which have been successfully used to multiplex a lot of users in the same cell while providing protection from multi-user interference are extremely inefficient.

There are many methods to resolve the collision problem from signal separation techniques and various medium access protocols. In [1], Tsatsanis first proposed a scheme called network-assisted diversity multiple accesses (NDMA) which exploits network diversity to separate the collided packets. Subsequently some of improved schemes were proposed. However, all these methods just only get the better throughput through the different retransmission schemes. For example, Rui Lin proposed a new wireless network medium access protocol based on the cooperation in [2].Dr. WeiJi presented a novel medium access scheme to deal the unfairness selection of the relay[3].

The drawbacks of these schemes are not only that they need to detect the channel information perfectly, but also the length of the ID sequences is so long which reduces the efficiency of transmission. If the channel varies fast, the receiver must detect the channel frequently. The result of the channel detection also decides the performance ofmaximum likelihood (ML) and zero forcing(ZF). So if the detection of the channel is not perfect, the performance of the decoder must be worse. In addition, these schemes detect the active users based on training sequences embedded in each user's packet head. In order to make the detection problem tractable, those training (or ID) sequences are orthogonal to each other [8].The orthogonality of the ID sequences makes the system sensitive to synchronizationand multipath effects. On the other hand, the length of the ID sequences is the same as the number of the users, so if there are a number of users in the system, the longer overhead may be substantial which has a negative impact on the bandwidth that carries the packet payload [10].

To overcome these drawbacks, there have been many considerable researches on blind and semi-blind separation in recent years. The concept of ICA is a relatively effective method. Independent Component Analysis (ICA) was first used in the universe of neural network model in the 80th of 20 century. Until 90th, some research groups bring in some successful methods, such as demo of the cocktail party problem. ICA can find every people's voice wave from the mixture signals. A.J.Bel and T.J.Sejnowski presented their methods based on Infomax [4], [5]. It is further detailed by the method called natural gradient method which is devised by S.I.Amari and his colleagues, and established the relation with the MLand Cichocki- Unbehauen method. Some years later, JuhaKarhunen proposed the fix point ICA method [6],[7].This method makes much contribution on theproblem of the large-scale applications.

This paper proposes a method which combinesthe retransmission scheme with ICA to resolve the collision problem and a new method to design the ID sequences to resolve the problems of ICA. Because every user's signal is independent with each other and non-Gaussian distribution, all these provide the conditions for the application of ICA. This algorithm converges very fast, and can solve the collision problem when there are many independent sources. Because the algorithm doesn't need to set the learning rate and any other parameters, so it is much robust and simple. Through searching the direction of the maximum nongausssinty of the received mixture signal matrix, ICA can separate the mixture signals without

the knowledge of the channel coefficients; In addition, the length of ID sequences is only $\log _{2}(J)+1$ ( $\mathrm{J}$ is the number of the users in the system).

The rest of the paper is organized as follows. In Section II, we describe the system model. The algorithm description and a new method that devise the ID sequences are introduced in Section III. The simulations and analysis are carried out in Section IV and we put conclusions in Section V.

\section{SYSTEM MODEL}

Fig. 1 shows the model of the retransmission model based on ICA. In this paper we consider a wireless cellular network, 
where there are $\mathrm{J}$ users. Every nodesis equipped with only one antenna.The system is slotted and every user transmits a packet consisting of $\mathrm{N}$ symbols in one slot. Once the collision is detected, the system enters a retransmission epoch.Assume that $\mathrm{K}(K \leq J)$ users are collided in the nth slot. At this time all the nodes and the destination will know the number of the collision users, and then the BS will send a control bit to all users in the system indicating the beginning of the retransmission epoch. In the following $\mathrm{K}-1$ slots, the collision users retransmit the packets which they transmitted during slot $\mathrm{n}$ (collision slot)and the other users which don't send the data during slot $n$ keep silence during this epoch. Fig.2 shows the two users model. We can see that the collision happens when user 1 anduser 2 send the data simultaneous in slot N. In the next slot, two collision users will retransmit their packets which send during slot $\mathrm{n}$.

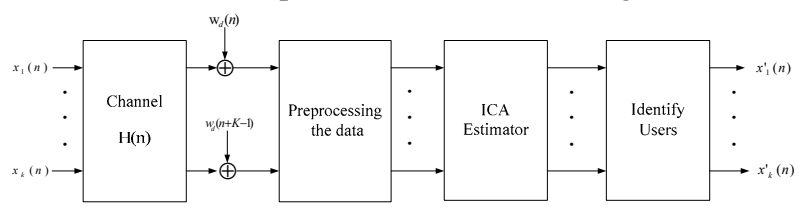

Figure 1. Multiple Packet Retransmission Scheme Based On ICA

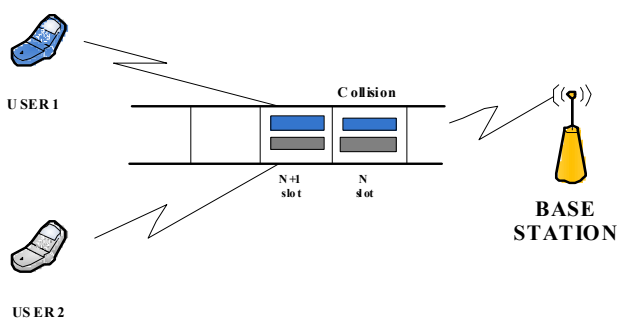

Figure 2. Two users' retransmission

In this paper, we consider a fast-fading channel. The mixture signals in the receiver can be expressed by the following model:

$$
\mathbf{Y}_{K \times N}=\mathbf{H}_{K \times K} \mathbf{X}_{K \times N}+\mathbf{W}_{K \times N}
$$

Where $\mathbf{Y}=\left[y_{N}(n), \ldots, y_{N}(n+K-1)\right]^{T}$ denotes the matrix of the observe signals during $\mathrm{K}$ slots at the base station, $y_{N}(n)$ consists of a mixture of collision data during slot $\mathrm{n}$, which has $\mathrm{N}$ bits; $\mathbf{X}=\left[x_{N, 1}(n), \ldots, x_{N, K}(n)\right]^{T}$ denotes the data of $\mathrm{K}$ collision users in slot $\mathrm{n}, x_{N, i}(n)$ is the ith user's data; $\mathbf{W}=\left[w_{N}(n), . ., w_{N}(n+K-1)\right]_{\text {denotes the noise matrix; During }}$ the $\mathrm{K}$ slots, the matrix of the channel coefficients between the collision users and BS is expressed as:

$$
\mathbf{H}=\left[\begin{array}{ccc}
a_{1 d}(n) & \cdots & a_{K d}(n) \\
\vdots & \ddots & \vdots \\
a_{1 d}(n+K-1) & \cdots & a_{K d}(n+K-1)
\end{array}\right]
$$

Where $a_{K d}(n)$ is the channel gain between the Kth user and the BS during slot $\mathrm{n}$.
Because the users' signals distribute non-Gaussian and are independent with each other, and the channel coefficients matrix is full rank, the ICA can be used to separate the signals. Before the application of the ICA algorithm, we preprocess the data through centering and whitening to make it a zero-mean and unit-variance variable.

Centering

$\mathbf{Y}^{\prime}=\mathbf{Y}-E[\mathbf{Y}]$

Whitening

$$
\mathbf{Y}^{*}=\mathbf{E D}^{-1 / 2} \mathbf{E} \mathbf{Y}^{\prime}=\widetilde{\mathbf{H}} \mathbf{X}
$$

Where $\mathrm{E}$ is the orthogonal matrix of eigenvectors of $E\left\{\mathbf{Y}^{\prime} \mathbf{Y}^{\prime T}\right\}$

eigenvalues, $\mathbf{D}=\operatorname{diag}\left(d_{1}, \ldots, d_{n}\right)$.The utility of whitening resides in the fact that the new mixing matrix $\widetilde{\mathbf{H}}$ is orthogonal. Then we use the separated matrixP to make a linear change on $\mathbf{Y}^{*}$. So we can get a K-dimensions output vectorZ. Our goal is to make $Z$ approach to the source signals, being the estimation of the independent components $\mathrm{X}$,

$$
\begin{aligned}
& \mathbf{Z}=\mathbf{P}^{\mathrm{T}} \mathbf{Y}^{*}=\mathbf{P}^{\mathrm{T}} \widetilde{\mathbf{H}} \mathbf{X}=\widehat{\mathbf{X}} \\
& \text { Where } \mathbf{P}=\left[p_{1}, p_{2}, \ldots, p_{k}\right] \text { is the inverse matrix of } \widetilde{\mathbf{H}} .
\end{aligned}
$$

\section{ALGORITHM DESCRIPTION}

\section{A. Algorithm introduction}

From the information theory: A Gaussian variable has the largest entropy among all random variables of equal variance. To obtain a measure of non-Gaussian that is zero for a Gaussian variable and always nonnegative, we should use the negentropy which can measure the independence among the signals. The maximum of the output negentropy means that the output signals are independent with each other, so we can separate the mixture signals. Therefore, the negentropy is often used as the target function of the blind separation. The expression of the negentropy is

$$
J(Z) \approx \sum_{i=1}^{K} a_{i}\{E[G(Z)]-E[G(V)]\}^{2}
$$

Where $a_{i}$ are some positive constants, and $\mathrm{V}$ is a Gaussian variable with zero mean and unit variance. The variable $\mathrm{Z}$ is assumed to be of zero mean and unit variance, and the function Gis anonquadratic function.

In the case where only one nonquadratic function Gis used, the approximation becomes

$$
J(Z) \propto[E\{G(Z)\}-E\{G(V)\}]^{2}
$$

Here we choose $G(u)=u^{4}$. Then the approximation function of negentropy is expressed as:

$$
J\left(\mathbf{P}^{\mathrm{T}} \mathbf{Y}^{*}\right) \propto\left[E\left\{G\left(p_{i}{ }^{\mathrm{T}} \mathbf{Y}^{*}\right)\right\}-E\{G(V)\}\right]^{2}
$$

When negentropy $J\left(\mathbf{P}^{\mathrm{T}} \mathbf{Y}^{*}\right)$ is maximal, it means that the ith source signal is separated. It notes that the maxima of the 
approximation of the negentropy of $p_{i}{ }^{\mathrm{T}} \mathbf{Y}^{*}$ are obtained at certain optima of $E\left[p_{i}^{T} \mathbf{Y}^{*}\right]$. The optima of $E\left[p_{i}^{T} \mathbf{Y}^{*}\right]$ under the constraint $E\left\{\left(p_{i}^{\mathrm{T}} \mathbf{Y}^{*}\right)^{2}\right\}=\left\|p_{i}\right\|^{2}=1$ are obtained at points:

$$
E^{\prime}\left[G\left(p_{i}^{T} \mathbf{Y}^{*}\right)\right]=E\left[\mathbf{Y}^{*} g\left(p_{i}^{T} \mathbf{Y}^{*}\right)\right]=0
$$

Here $\mathrm{g}(\mathrm{x})$ is the differential of $\mathrm{G}(\mathrm{x})$. We can solve this by Newton's method

$$
\begin{aligned}
p_{i}{ }^{\prime} & =p_{i}-\frac{E\left[\mathbf{Y}^{*} g\left(p_{i}^{T} \mathbf{Y}^{*}\right)\right]}{E\left[\mathbf{Y}^{*} \mathbf{T} \mathbf{Y}^{*} g^{\prime}\left(p_{i}^{T} \mathbf{Y}^{*}\right)\right]} \\
& =p_{i}-\frac{E\left[\mathbf{Y}^{*} g\left(p_{i}^{T} \mathbf{Y}^{*}\right)\right]}{E\left[g^{\prime}\left(p_{i}{ }^{T} \mathbf{Y}^{*}\right)\right]}
\end{aligned}
$$

Equation (10) can be further simplified by multiplying both sides by $E\left[g^{\prime}\left(p_{i}^{T} \mathbf{Y}^{*}\right)\right]$, then

$$
p_{i}^{\prime} E\left[g^{\prime}\left(p_{i}^{T} \mathbf{Y}^{*}\right)\right]=p_{i} E\left[g^{\prime}\left(p_{i}^{T} \mathbf{Y}^{*}\right)\right]-E\left[\mathbf{Y}^{*} g\left(p_{i}^{T} \mathbf{Y}^{*}\right)\right]
$$
obtain:

Substituting $p_{i}^{*}=-p_{i}{ }^{\prime} E\left[g^{\prime}\left(p_{i}^{T} \mathbf{Y}^{*}\right)\right]$ into (11) and we can

$$
p_{i}^{*}=E\left[\mathbf{Y}^{*} g^{\prime}\left(p_{i}^{T} \mathbf{Y}^{*}\right)\right]-E\left[g^{\prime}\left(p_{i}^{T} \mathbf{Y}^{*}\right)\right] p_{i}
$$

Then renormalize $p_{i}{ }^{*}$ :

$$
p_{i}^{+}=p_{i}^{*} /\left\|p_{i}^{*}\right\|
$$

If the algorithm is not convergence, the above process will be repeated. Because the algorithm needs to estimate $\mathrm{K}$ vectors (from $p_{1}$ to $p_{k}$ ), to prevent different vectors from converging to the same maxima, it decorrelates the outputs $p_{1}^{T} \mathbf{Y}^{*}, \ldots, p_{k}{ }^{T} \mathbf{Y}^{*}$ after every iteration. In this paper we use a symmetric decorrelation

$$
\mathbf{P}=\left(\mathbf{P} \mathbf{P}^{\mathbf{T}}\right)^{-1 / 2} \mathbf{P}
$$

The process of the algorithm is depicted as follow:

Select $\mathrm{m}$, the number of the independent components.

Initiate all $p_{i}, i=1,2, \ldots, m$. Every $p_{i}$ has a ordinary norm. The matrix P should be decorrelated at the 4th method.

$$
\begin{aligned}
& \text { For } \mathrm{i}=1, \ldots, \mathrm{m}, \text { refresh }{ }^{p_{i}}: \\
& p_{i} \leftarrow E\left[\mathbf{Y}^{*} g\left(p_{i}^{T} \mathbf{Y}^{*}\right)\right]-E\left[g^{\prime}\left(p_{i}^{T} \mathbf{Y}^{*}\right)\right] p_{i} . \\
& \text { Then decorrelate } \mathbf{P}=\left(p_{1}, \cdots, p_{m}\right)^{T}: \\
& \mathbf{P} \leftarrow\left(\mathbf{P P}^{\mathbf{T}}\right)^{-1 / 2} \mathbf{P}
\end{aligned}
$$

If the iteration is not convergence, then return 3.

From the above, it can be seen that the ICA method doesn't need to obtain the mixture matrix coefficients.

\section{B. User detection}

ICA has two problems hard to solve: First, it is difficult to identify the order of the independent components which means that we can't make sure the separated signals belong to which user. The reason is that both $\mathrm{H}$ and $\mathrm{X}$ being unknown, it can freely change the order of the terms in (1). So in the stage of user detection, ID sequences are needed to identify the users; Secondly, the sign of the separated signals may be changed, such as the information of user $i$ changing from $[1,-1,1,-1]$ into $[-1,1,-1,1]$ after the ICA processing.

In this paper, we design the new ID sequences for the problems of ICA. The length of the new ID sequences is only $\log _{2}(J)+1$ and every user's ID sequences are not to be orthogonal to the others. It overcomes the drawbacks of the traditional ID sequences. We use $\mathrm{m}\left(\mathrm{m}=\log _{2}(J)\right.$ ) binary numbers to express $\mathrm{J}$ users. Then we use the idea of the differential encoding to encode the $\mathrm{k}$ binary numbers. For example, there are 32 users in the system, and the 5th user's ID sequence is $(0,0,1,0,0)$ and we encode it by differential BPSK encoding $(1,1,1,-1,-1,-1)$, with the length $\log _{2}(J)+1$. Though the ICA changes the plus-minus of the separated signals, the relationship between the codes is not changed.

\section{Simulation}

First, we use two users model to see whether the ICA can separate the mixed signals. The channel matrix during two slots is

$$
H=\left[\begin{array}{cc}
0.5309 & 0.4076 \\
0.6544 & 0.8200
\end{array}\right]
$$

And the users' packets are encoded by BPSK. The SNR were $20 \mathrm{~dB}$.

From the Figure 3, it is obvious that ICA can completely separate the mixture signals. So the collision in the random access network is seem to be resolved by ICA.

We simulate the throughput of the proposed scheme in ZF、ICA、ML. Here the throughput of the

system is defined as:

$$
\text { throughput }=\frac{\text { successful received packets }}{\text { retransmission times }}
$$

The total number of users in the system is $\mathrm{J}=32$, and the users' ID sequences are encoded by differential encoding as above introduction. We define traffic load $\lambda$, as the number of packets that are fed into the network during a specific time slot. Every user's data is encoded byBPSK. The channel between eachuser and

the BS is Rayleigh fading and every packet contains 424bits. The simulation is under $\mathrm{SNR}=20 \mathrm{~dB}$ scenarios. We complete 2000 trials. In one trial, each user sends out the packet with probability $\lambda / J$.we set bit error rate is at most 0.02 and packets received at the AP with bit error rate higher than 0.02 are considered lost or corrupted. If the number of iteration of ICA is over 1000 times, we think the algorithm is false and the packet is lost.

We can see that the throughput of ICA method is better than the ZF method without the coefficients of the channel. Through the throughput of ICA is worse than ML, it is known that the complexity of ML is very high and the complexity of ICA is lower than ML. Fig. 5 shows the relation between the throughput and SNR, it expresses that 
the throughput is become lower as the SNR decreases. Here SNR is from $10 \mathrm{~dB}$ to $20 \mathrm{~dB}$.

Fig. 6 shows that the throughput of ICA and ZF at the data rate $\mathrm{R}=256 \mathrm{~kb} / \mathrm{s}$ and $\mathrm{R}=12 \mathrm{Mb} / \mathrm{s}$, we can see that when the data rate is $12 \mathrm{Mbps}$, the channel is slow varying channel, thus the channel coefficients are correlated. Both throughput decrease a little, but the result of ICA is still better than ZF.

From the above simulations, ICA algorithm can separate the mixture signals very well. Because the retransmission scheme itself do have some drawbacks, so the result of the ICA is a little bad. But we can see that the result of ICA is still better than ZF based on the same scheme.
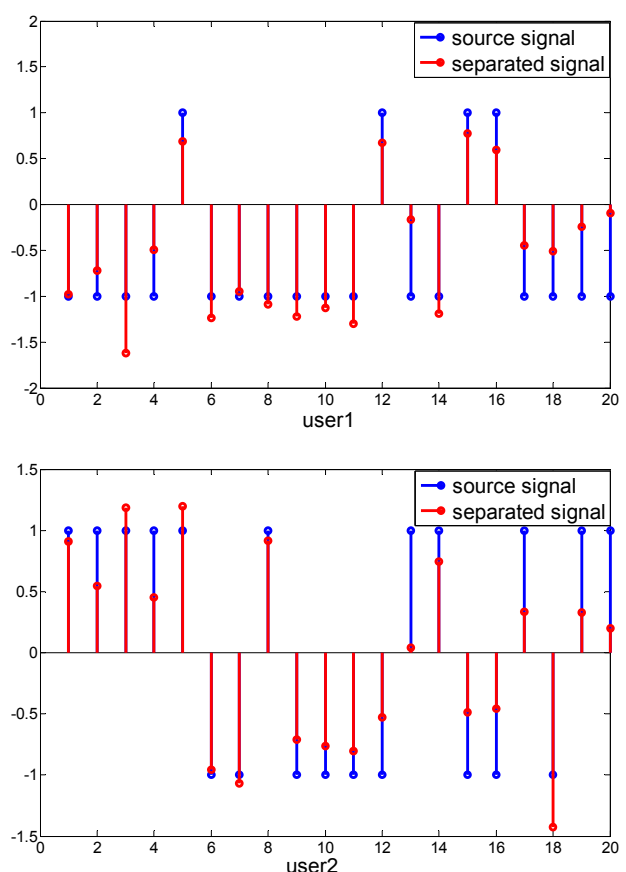

Figure 3. Two users model:source signals and separated signals

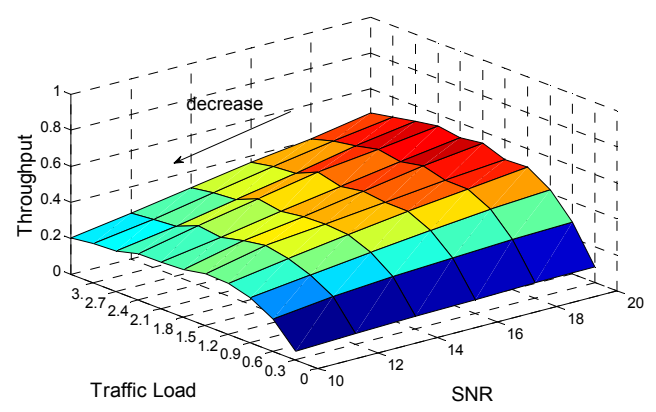

Figure 4. Throughput vs SNR

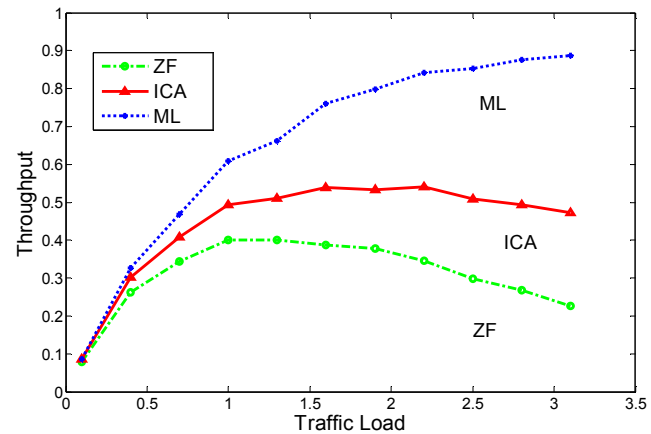

Figure 5. The throughput of the ZF, ICA, ML.R=256kbps,

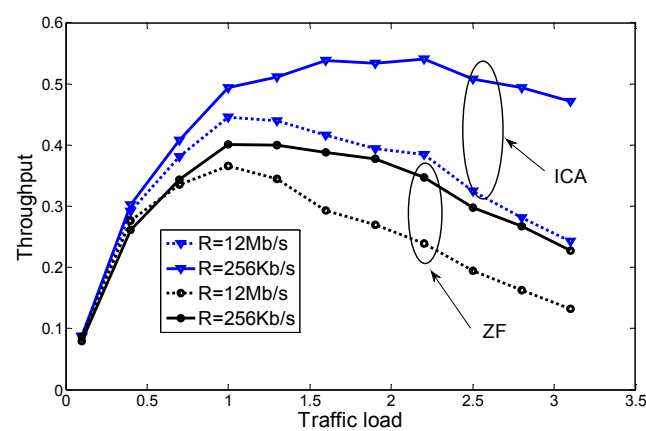

Figure 6. Throughput versus traffic load of ICA, ZF: R=12Mb/s and $\mathrm{R}=256 \mathrm{~kb} / \mathrm{s}$

\section{CONCLUSION}

In this paper, we present a new multiple packets retransmission scheme based on ICA and devise the new ID sequences. ICA can separate the mixture signals without the knowledge of the channel, so it can reduce the BS station complexity. According to the simulation results, the ICA method can get a better performance compared with ZF. The new ID sequences can improve the transmit efficiency and solve the problems of the ICA. So the proposed scheme is a fully blind random access technique with high performance.

\section{REFERENCES}

[1] M.K.Tsatsanis, R.Zhang, and S.Banerjee, Network-Assisted Diversity for Random Access Wireless Networks, IEEE Trans. Signal Process, vol. 48, pp. 702-711,Mar.2000

[2] Rui Lin and Athina P.Petropulu, A New Wireless Nerwork Medium Access Protocol Basedon Cooperation, IEEE Transactionson Signal Processing, vol, 53, No.12. December 2005.

[3] Ji Wei, "Communication and Information Systems". School of electronics, information and electrical engineering Shanghai Jiao Tong University, December 2008.

[4] A.J.Bell and T.J.Sejnowski. A non-linear information maximization algorithm that performs blind separation. In Advances in Neural Information Processing Systems 7 pages 467-474. The MIT Press, Cambridge, MA 1995.

[5] A.J.Bell and T.J.Sejnowski. An information maximization approach to blind separation and blind deconvolution. Neural Computation, 7: 1129-1159, 1995. 
[6] A.Hyvarinen and E.Oja.A fast fixed-point algorithm for independent component analysis.Neural Coputation, 9(7): 1483-1492, 1997.

[7] A.Hyvarinen. A family of fixed-point algorithms for independent component analysis.In proc.IEEE Int.Conf.on Acoustics, Speech and Signal Processing, pages 3917-3920, Munich, Germany, 1997.

[8] R. Zhang, N. D. Sidiropoulos, and M. Tsatsanis, "Collision Resolution in Packet Radio Networks Using Rotational Invariance Techniques", IEEE Trans. Com. submitted.

[9] M. Tsatsanis, R. Bang and S. Banerjee, "CollisionResolution Techniques for Wireless Random Access Networks without
Throughput Penalty", Proc. of IEEE 1998 International Conference on Universal Personal Communications 11CLIPC98). Florence. Italy, Oct. 5-9, 1998, pp. 1325-1329

[10] Ozgul, B.; Delic, H.; , "Blind collision resolution for mobile networks in fast-fading channels," Communications, 2003. ICC '03. IEEE International Conference on , vol.2, no., pp. 1228- 1232 vol.2, 11-15 May 2003 